\title{
Expression and localization of the immunophilin FKBP51 in colorectal carcinomas and primary metastases, and alterations following oxaliplatin-based chemotherapy
}

\author{
DEBORAH ROTOLI ${ }^{1,2 *}$, MANUEL MORALES $^{3,4 *}$, MARÍA DEL CARMEN MAESO ${ }^{5}$, \\ MARÍA DEL PINO GARCÍA ${ }^{6}$, ARACELI MORALES ${ }^{7}$, JULIO ÁVILA ${ }^{1}$ and PABLO MARTÍN-VASALLO ${ }^{1}$
}

\begin{abstract}
${ }^{1}$ Developmental Biology Laboratory, UD- Biochemistry and Molecular Biology and Centre for Biomedical Research of the Canary Islands, La Laguna University, La Laguna, 38206 Tenerife, Spain; ${ }^{2}$ National Research Council, Institute of Endocrinology and Experimental Oncology, I-80131 Naples, Italy; ${ }^{3}$ Service of Medical Oncology, University Hospital

Nuestra Señora de Candelaria, 38010 Santa Cruz de Tenerife; ${ }^{4}$ Unit of Medical Oncology, Hospiten ${ }^{\circledR}$ Hospitals, Santa Cruz de Tenerife, 38001 Tenerife; ${ }^{5}$ Service of Pathology, University Hospital Nuestra Señora de Candelaria, 38010 Santa Cruz de Tenerife; ${ }^{6}$ Laboratory of Biopathology, Hospiten ${ }^{\circledR}$ Hospitals, 38010 Tenerife; ${ }^{7}$ Department of

Physiology, Institute of Biomedical Technologies, School of Medicine and Centre for Biomedical Research of The Canary Islands, University of La Laguna, La Laguna, 38071 Santa Cruz de Tenerife, Spain
\end{abstract}

Received September 28, 2015; Accepted April 29, 2016

DOI: $10.3892 / \mathrm{ol} .2016 .4772$

\begin{abstract}
The immunophilin FK506-binding protein 5 (FKBP51) is a scaffold protein that serves a pivotal role in the regulation of multiple signaling pathways, integrating external and internal stimuli into distinct signal outputs. In a previous study, we identified several genes that are significantly up- or downregulated in the peripheral white cells (PWCs) of colorectal adenocarcinoma (CRC) patients undergoing oxaliplatin-based chemotherapy. In our screening, FKBP51 gene expression was downregulated following chemotherapy. In order to determine whether this alteration in gene expression observed in PWCs may be detected at the protein level in tumors and metastases following the administration of adjuvant chemotherapy, an immunohistochemical analysis of FKBP51 in CRC and primary metastasis tissues was performed. The present study confirmed the downregulation of FKBP51 gene expression elicited by chemotherapy with folinic acid (leucovorin), fluorouracil and oxaliplatin in metastasized liver tissue that had been resected after the oxaliplatin-based chemotherapy, compared with tissue section samples of CRC from patients (prior to antineoplastic
\end{abstract}

Correspondence to: Professor Pablo Martín-Vasallo, Developmental Biology Laboratory, UD- Biochemistry and Molecular Biology and Centre for Biomedical Research of the Canary Islands, La Laguna University, Av. Astrofísico Sánchez, La Laguna, 38206 Tenerife, Spain

E-mail:pmartin@ull.es

*Contributed equally

Key words: colorectal cancer, oxaliplatin, FK506-binding protein 5, immunophilin treatment). Furthermore, the results indicated that, in CRC tissue sections, the expression of FKBP51 protein is associated with an immature phenotype of stromal fibroblasts and with the epithelial-to-mesenchymal transition (EMT) phenotype, suggesting a role for this protein in the EMT process in CRC. Finally, the observation that only certain cells of the stroma express FKBP51 protein suggests a potential role for this immunophilin as a stroma cell subtype marker.

\section{Introduction}

In the Western world, colorectal cancer (CRC) is the fourth leading cause of cancer-associated mortality (1), and $>95 \%$ of colorectal cancers are adenocarcinomas. The main risk factors are genetic factors (family history), a low fiber and high fat diet, and smoking (2). Following curative surgery alone, the percentage of patients that subsequently relapse and succumb to metastatic disease ranges from 40 to $50 \%$. This percentage falls to $33 \%$ when patients receive postoperative adjuvant treatment with 5-fluorouracil (5-FU) and leucovorin, and to $23 \%$ when the platinum-containing compound oxaliplatin is added to this treatment [FOLFOX: folinic acid (leucovorin), 5-FU and oxaliplatin] $(2,3)$.

Unfortunately, despite the positive results obtained by the combination protocols of oxaliplatin with fluoropyrimidines (e.g. 5-FU) and folinic acid (leucovorin) in disease-free survival of stage II or III colon cancer (3), unwanted side effects develop that can affect, to various degrees, the quality of life of the patients. Several side effects have been reported for oxaliplatin, which include gastrointestinal toxicity, moderate hematological toxicity, hypersensitivity and neurological toxicity. This unpredictable neural toxicity has quite unique features and determines the dose-limiting toxicity of oxaliplatin (4). 
The identification of biomarkers that could predict the onset of these secondary effects would be of great value in preventing long-term toxicity or permanent damage in patients at risk. Recent research from our laboratory has led to the identification of several genes that are significantly up- or downregulated in peripheral white cells (PWCs) of CRC patients following oxaliplatin-based chemotherapy treatment (5). In our screening, the expression levels of the gene encoding for the immunophilin FK506-binding protein 5 (FKBP51) were 2.76-times lower [3,812 (pre) vs. 1,383 (post)] in PWCs after 3 cycles of oxaliplatin-based chemotherapy (5).

FKBP51 protein plays multiple roles in the regulation of a variety of signaling pathways, and has altered expression levels in many different tumor types. By regulating steroid receptor maturation, as well as the Akt and nuclear factor $\kappa \mathrm{B}$ signaling pathways, FKBP51 is important in tumorigenesis and in the response to chemotherapy (6-13). FKBP51 belongs to a superfamily of peptidyl-prolyl isomerases (PPIs), which also includes FKBP52 and the cyclosporine A-binding protein cyclophilin-40. FKBP51 is a $51 \mathrm{kD}$ FK506-binding protein with a C-terminal tetratricopeptide repeat (TPR) domain, and an N-terminal FK1 domain responsible for PPIase activity, which catalyzes the cis-trans conversion of prolyl peptide bonds within target proteins (14). Through the TPR domain, FKBP51 binds to heat shock protein 90 (HSP90) complexes, such as those associated with steroid hormone receptors. The mechanisms underlying the regulation of steroid hormone receptor signaling by this immunophilin and its physiological roles in endocrine-related processes are very well studied and will be further discussed in the discussion section.

Research in this field has led to the identification of FKBP51 as a potential therapeutic target for several endocrine-related diseases, such as metabolic and stress-related diseases, prostate cancer and breast cancer (13). Diseases associated with this protein include major depressive disorder and glucocorticoid resistance (Gene Ontology annotations). FKBP51 is a ubiquitous protein expressed in the cytoplasm, nucleus and mitochondria (14). Nuclear-mitochondrial shuttling is triggered by oxidative stress: The protein translocates to the nucleus upon the onset of oxidative stress to protect the cells from stress damage (7).

Recently, a role for the immunophilins FKBP51 and FKBP52 in regulating microtubules has been suggested, acting via their interaction with $\tau$ proteins (15). Regulation of microtubule dynamics by FKBPs has been associated with neurite outgrowth (16). Furthermore, FKBP51 has been identified as a regulator of cell death in response to gemcitabine and cytarabine treatment: High levels of FKBP51 expression were associated with sensitivity, while low levels of expression were associated with resistance to these drugs (17).

Several studies also link FKBP51 to cell proliferation and cancer. It has been demonstrated, for example, that it plays a role in negatively regulating the Akt pathway. Acting as a scaffold protein, FKBP51 promotes the interaction of Akt and PHLPP, a phosphatase that specifically dephosphorylates Akt at Ser473 and inhibits its activity (9). Recently it has been demonstrated that FKBP51 is key in promoting the activation of genes involved in melanoma progression (11), and modulates the transforming growth factor $\beta$ (TGF- $\beta$ ) signal in malignant melanocytes, increasing the tumor-promoter potential of TGF- $\beta$ (12). FKBP51 expression is also decreased in pancreatic cancer tissues and in numerous cancer cell lines.

In the current study, an immunohistochemical (IHC) analysis of FKBP51 in CRC tissue sections (namely before antineoplastic therapy) and primary metastases (resected after oxaliplatin-based chemotherapy) was performed in order to determine whether the alteration in FKBP51 gene expression observed in PWCs can be detected at the protein level, the nature of their role in tumoral physiopathology, and whether these alterations have any prognostic implications.

\section{Materials and methods}

Patients. The study was approved by the Ethics Committee of La Laguna University (La Laguna, Spain) and the Ethics Committee of Nuestra Señora de Candelaria University Hospital (HUNSC; Santa Cruz de Tenerife, Spain). All patients signed informed consent for diagnosis and research on tissue specimens, prior to entering the study. All subjects were treated with FOLFOX chemotherapy as follows: Day 1 , oxaliplatin $\left[100 \mathrm{mg} / \mathrm{m}^{2}\right.$ intravenous (i.v.) over $2 \mathrm{~h}$ ], leucovorin calcium $\left(400 \mathrm{mg} / \mathrm{m}^{2}\right.$ i.v. over $2 \mathrm{~h}$ ); followed by $5-\mathrm{FU}\left(400 \mathrm{mg} / \mathrm{m}^{2}\right.$ i.v. bolus) and by $5-\mathrm{FU}$ $\left(2,400 \mathrm{mg} / \mathrm{m}^{2}\right.$ i.v. over $\left.46 \mathrm{~h}\right)$, every 14 days, days, and no patient underwent previous CT scans or received radiation therapy. The patients were treated between October 2010 and July 2015.

Several (between 10 and 15) mounted slides of paraffin-embedded tissue samples ( $5 \mu \mathrm{m}$ thick; colon adenocarcinoma and metastasized liver and lung from selected patients) obtained during resection and clinical data were collected from 33 patients (16 males and 17 females), aged between 38 and 76 years, from the reference medical areas of HUNSC.

Antibodies. The following antibodies were used: Rabbit polyclonal antibody (pAb) against FKBP51 (\#ab46002; Abcam, Cambridge, UK; dilution, 1.25:100); mouse monoclonal antibody against proliferating cell nuclear antigen (PCNA; clone PC10; \#1486 772, Roche Diagnostics Deutschland GmbH, Mannheim, Germany; dilution, 1:100); fluorescein isothiocyanate (FITC)-conjugated goat pAb against rabbit IgG (\#F9887; Sigma-Aldrich, St. Louis, MO, USA; dilution, 1:200); goat pAb against mouse IgG (DyLight ${ }^{\circledR}$ 650; \#ab97018; Abcam, dilution, 1:100); biotin-conjugated goat pAb against rabbit $\operatorname{IgG}(\mathrm{H}+\mathrm{L})$ (\#31820; Thermo Fisher Scientific, Inc., Waltham, MA, USA; dilution, 1:300).

IHC. Immunoperoxidase staining of formalin-fixed, paraffin-embedded tissue sections was performed using an ordinary avidin-biotin method. Briefly, $5 \mu \mathrm{m}$-thick tissue sections were deparaffinized in xylene and hydrated in graded alcohol. Heat-induced epitope retrieval was achieved by heating samples in sodium citrate buffer $\mathrm{pH} 6.0$ at $120^{\circ} \mathrm{C}$ for $10 \mathrm{~min}$ in an autoclave. After non-specific sites were blocked with $5 \%$ non-fat dry milk in Tris-buffered saline (TBS) for $1 \mathrm{~h}$ at room temperature, endogenous biotin was blocked using an Avidin/Biotin Blocking Kit (Vector Laboratories, Inc., Burlingame, CA, USA). Primary antibody against FKBP51 (1.25:100) was applied to slides overnight at $4^{\circ} \mathrm{C}$. Biotin-conjugated anti-rabbit secondary antibody was incubated for $2 \mathrm{~h}$ at $37^{\circ} \mathrm{C}$ at a dilution of 1:300. To block endogenous peroxidase activity, slides were incubated with $3 \%$ hydrogen peroxidase in methanol 

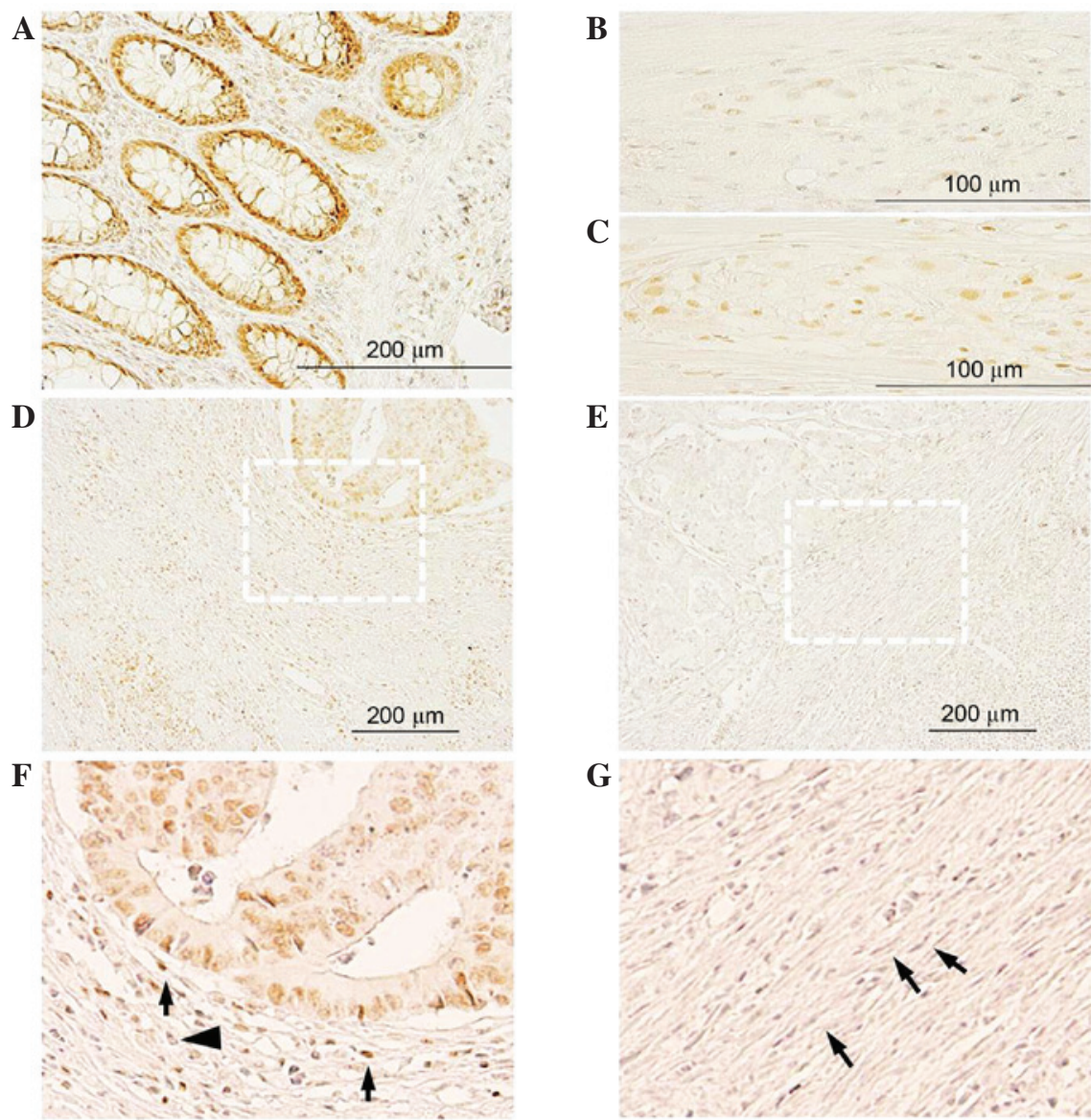

$\mathbf{E}$
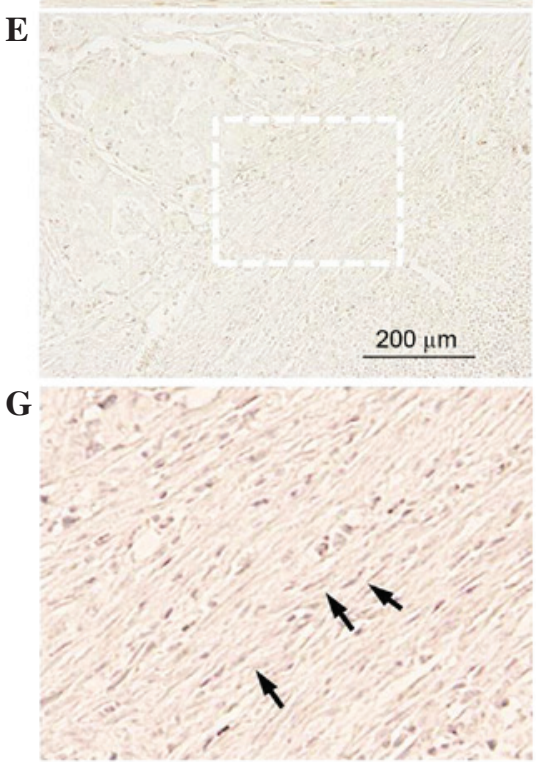

Figure 1. (A and B) FKBP51 expression in healthy colon: (A) Positive staining in enterocytes and cells of the lamina propria; (B) in healthy colon, few cells in Auerbach's plexus exhibit a weak signal. (C) By contrast, in colorectal cancer tissue sections, positive staining is observed in several cells of the plexus. (D-G) FKBP51 expression in colon adenocarcinoma: Tumor cells and inflammatory and fibrous stromal cells exhibit variable signals, from (D) strongly positive to (E) absent. (F) Magnification of (D), showing immature phenotype of stromal fibroblasts surrounding a lesion with positive FKBP51 cells. (G) Magnification of (E), showing mature phenotype of stromal fibroblasts surrounding a lesion with cells completely negatively expressing FKBP51. FKBP51, FK506-binding protein 5.

for 15 min. A Pierce ABC Peroxidase Staining Kit (Thermo Fisher Scientific, Inc.) was used to amplify the specific antibody staining. Concentrated 3,3'-diaminobenzidine Substrate (\#IHC-101F; Bethyl Laboratories, Inc., Montgomery, TX, USA) was used to visualize IHC reactions. Samples incubated without primary antibodies were used as negative controls. Slides were counterstained with Harris Hematoxylin Solution DC (Panreac Quimica SLU, Barcelona, Spain) to visualize cell nuclei. Slides were mounted with Eukitt (Panreac Quimica SLU). An optical light microscope (BX50, Olympus Corporation, Tokyo, Japan) was used to visualize immunostaining results.

Image analysis and statistics. For semi-quantitative image analysis, the open resource digital image analysis software ImageJ was used, implemented with the IHC Profiler plug-in developed by Varghese et al (18), which creates a pixel-by-pixel analysis profile of a digital IHC image, and further assigns a score in a four tier system: High positive (pixel intensity range, 0-60), positive (pixel intensity range, 61-120), low positive (pixel intensity range, 121-180), negative (pixel intensity range, 181-235). All images were captured at the same magnification (40x) and with the same levels of contrast and brightness. Pearson's Correlation Coefficient and Student's $t$-test were performed using SPSS version 20 software (IBM SPSS, Madrid, Spain) in order to estimate the reliability of the study.
Double immunofluorescence simultaneous staining. Following the deparaffinization, hydration and heat-induced epitope retrieval procedures (as described), slides were incubated with 5\% bovine serum albumin (catalog no. A9647; Sigma-Aldrich, St. Louis, MO, USA) and 1\% Triton X-100 in TBS to block non-specific sites. Tissue sections were then incubated simultaneously with a mixture of two distinct primary antibodies (rabbit anti-FKBP51 and mouse anti-PCNA) overnight at $4^{\circ} \mathrm{C}$, at concentrations of 1:50 and 1:100, respectively. Slides were then incubated for $1 \mathrm{~h}$ at room temperature with a mixture of two secondary antibodies (FITC-conjugated anti-rabbit and DyLight $^{\circledR}$ 650-conjugated anti-mouse). Slides were mounted with ProLong ${ }^{\circledR}$ Diamond Anti-fade Mountant with DAPI (Molecular Probes; Thermo Fisher Scientific, Inc., Eugene, Oregon, USA) to visualize cell nuclei. Slides were analyzed using a confocal microscope (FV1000, Olympus Corporation).

\section{Results}

IHC analysis of FKBP51 expression in colon tissue samples from $C R C$ patients. In healthy colon and in the apparently healthy region of the CRC tissue sections (Fig. 1), intestinal glands exhibited intense positive FKBP51 nuclear staining in enterocytes and in cells of the lamina propria (Fig. 1A). In healthy colon, few cells in the myenteric plexus exhibited a 

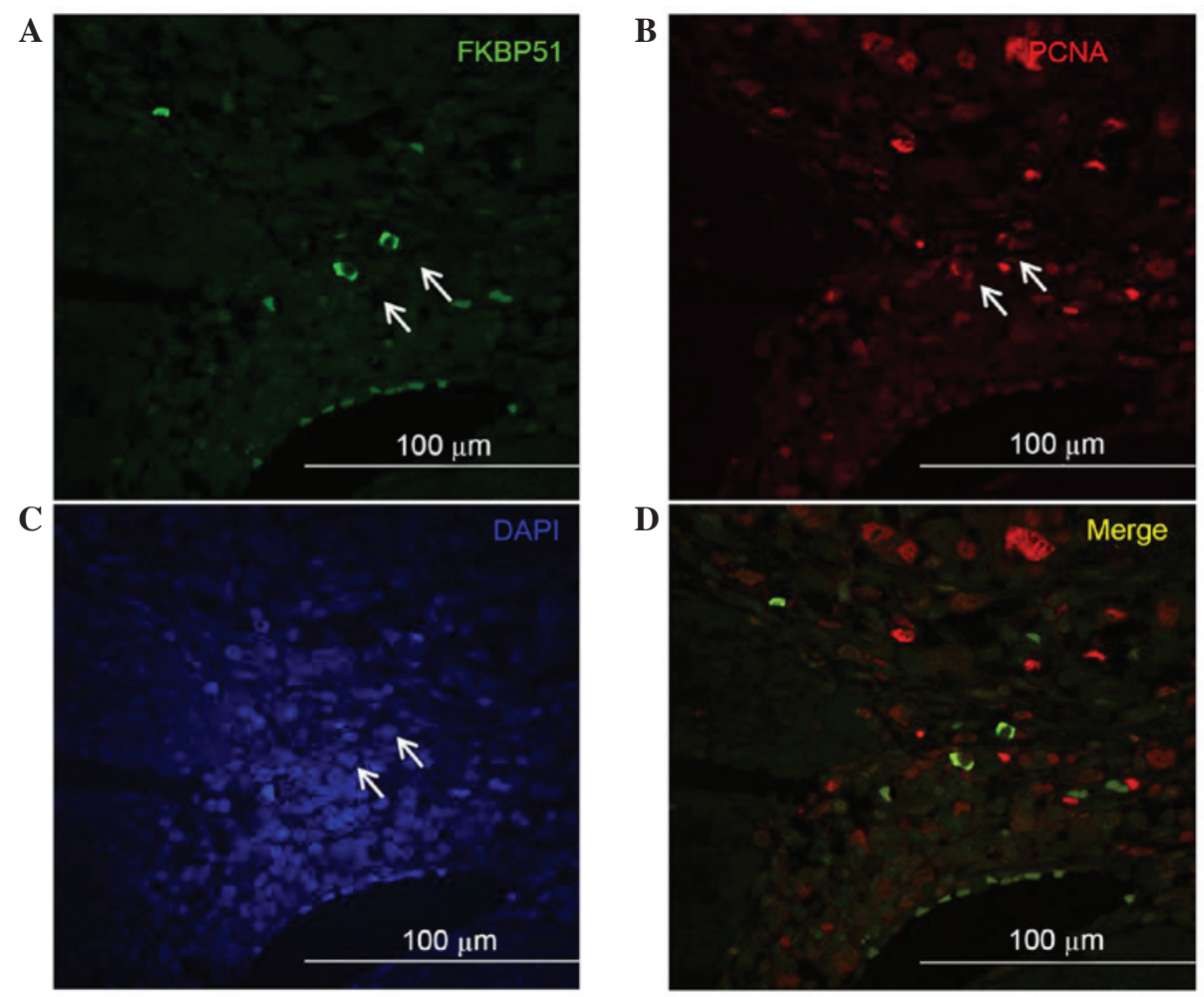

Figure 2. Double immunofluorescence imaging on colorectal cancer tissue sections. Confocal images of (A) FKBP51 (green) and (B) PCNA (red) expression in stromal cells; only certain cells in the stroma co-express FKBP51 and PCNA (arrows). (C) Nuclei were visualized by DAPI. (D) Merged image of (A) and (B). FKBP51, FK506-binding protein 5; PCNA, proliferating cell nuclear antigen.

weak signal (Fig. 1B), while in CRC tissue sections, several cells in the plexus were strongly positive (Fig. 1C).

In colon adenocarcinoma tissue sections, FKBP51 protein was localized in the cytoplasm and/or nucleus of tumor cells, as well as in inflammatory and fibrous stromal cells surrounding the lesions (Fig. 1D-G). In certain areas of the section, a variable positive signal could be observed in tumor cells, while in other areas, no staining was detected. Notably, the phenotype of the connective tissue surrounding the lesions appeared variable: In those areas where no immunophilin expression was observed in tumor and stromal cells, stromal fibroblasts exhibited a mature phenotype, with thin, wavy and small spindle cell morphology (Fig. 1E and G, arrows); by contrast, in those areas where positive FKBP51 immunostaining could be observed in tumor and stromal cells, fibroblasts exhibited an immature phenotype, with large, puffy, spindle-shaped morphology (Fig. 1F). An increased microvessel density and enhanced infiltration of tumor-associated macrophages was also observed in the connective tissue surrounding FKBP51-positive lesions (Fig. 1F).

In the stroma surrounding tumor nests, the expression of FKBP51 was variable, with cells exhibiting a strong positive signal (Fig. 1F, arrow) and others completely negative (Fig. 1F, arrowhead).

Double immunofluorescence experiments to detect FKBP51 and PCNA, the clamp subunit of DNA polymerase and marker of $S$ phase of cell cycle (19), revealed that, among the stromal cells expressing PCNA, only a few coexpressed FKBP51 (Fig. 2). This suggests a potential role for this immunophilin protein as a marker of specific subtypes of stromal cells; however, further studies are needed to assess this hypothesis.
IHC analysis of FKBP51 expression in metastasized liver tissue samples from CRC patients. In the overall sections of healthy liver (Fig. 3C) and in the apparently healthy part of metastasized liver (Fig. 3D), there were areas with a more intense signal and well-delimited areas in which FKBP51 protein expression was weak or absent. Intense staining could be observed in the nuclei of hepatocytes lining the edge of the connective tissue capsule (Glisson's capsule) (Fig. 3A, arrows). By contrast, in metastasized liver, this nuclear signal was fainter (Fig. 3B, arrow). In metastases, the signal appeared faint or absent, while several of the inflammatory fibrous stroma cells exhibited a strong signal (Fig. 3E).

IHC analysis of FKBP51 expression in metastasized lung tissue samples from CRC patients. In healthy lung tissue, positive FKBP51 staining was observed in macrophages and endothelial cells. In the respiratory mucosa, a strong signal was present in the nuclei and cytoplasm of ciliated cells (data not shown). In metastasized lung, strong positive staining could be observed in macrophages (Fig. 4A, black arrow), in cells of the lamina propria, in endothelial cells and, to a lesser extent, in the nuclei of bronchial gland cells (Fig. 4C, arrowhead, thick arrow and thin arrow, respectively). In the bronchial epithelial cells, FKBP51 protein was localized to the nuclei and/or cytoplasm of ciliated cells; strong staining in the basal bodies of these cells was present (Fig. 4B, arrow). In the malignant area of the section, weakly positive or no staining was observed in tumor cells, while the signal appeared stronger in inflammatory and fibrous stromal cells surrounding the lesions (Fig. 4E and F). Fig. 4F shows the positive protein staining in several stromal cells between two metastases; the intracellular distribution 
A



$\mathbf{E}$

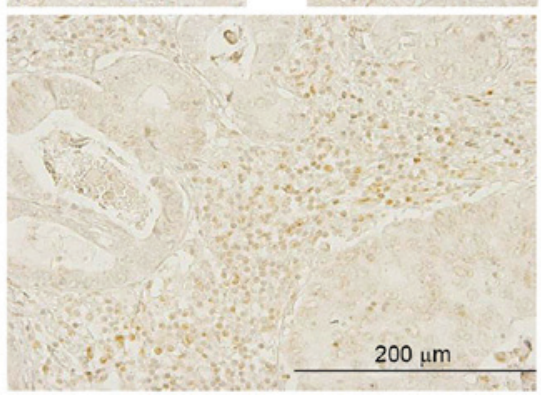

C

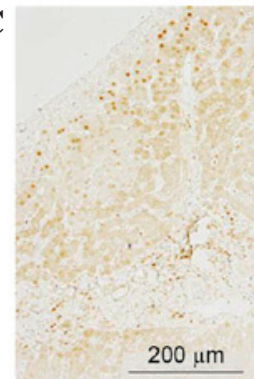

D

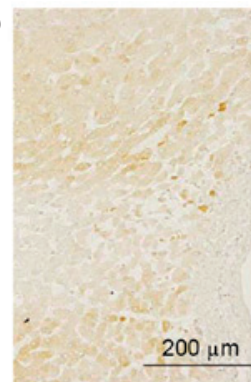

F

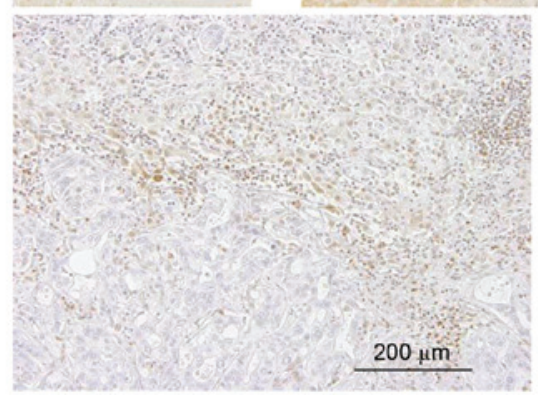

Figure 3. FKBP51 expression in healthy liver and in metastasized liver. Positive nuclear staining in hepatocytes lining the edge of Glisson's capsule (black arrows) was observed in (A) healthy and (B) metastasized liver tissue. Variable FKBP51 protein expression was present in (C) healthy liver and (D) apparently healthy parts of metastasized liver. (E and F) Inflammatory fibrous stroma exhibited positive staining in several cells, while in metastasis, the signal is (E) faint or (F) absent. FKBP51, FK506-binding protein 5.

A



C

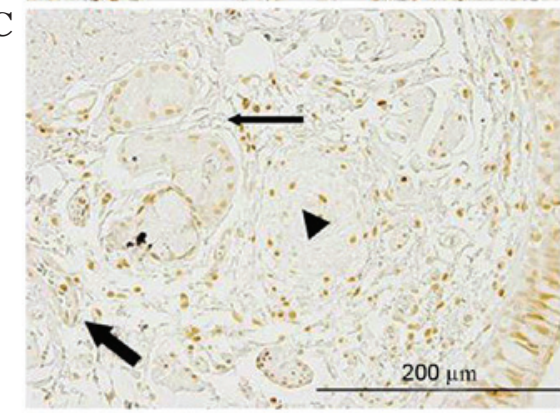

E



B



D

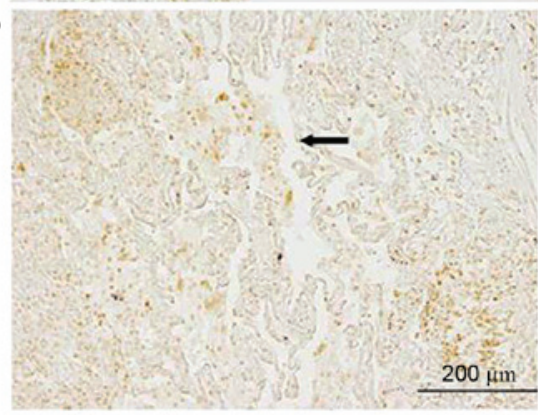

F



Figure 4. Anti-FK506-binding protein 5 immunostaining in metastasized lung tissue section. (A) Macrophages (arrow) and endothelial cells (arrowhead) exhibited strong positive signals. (B) Respiratory mucosa showed intense positive staining in the nuclei and cytoplasm of ciliated cells; intense staining in basal bodies of ciliated cells could be observed (arrow). (C) Strong positive staining was observed in cells of the lamina propria (arrowhead) and in endothelial cells (thick arrow); nuclei of bronchial gland cells exhibited a positive signal (thin arrow). (D) Clustered macrophages were visible (arrow). (E and F) Tumor cells exhibited weak positive staining, while a strong positive signal was observed in inflammatory and fibrous stromal cells surrounding the lesions. (F) The inset image (lower left) shows x100 magnification of a cell to illustrate further distribution detail. 


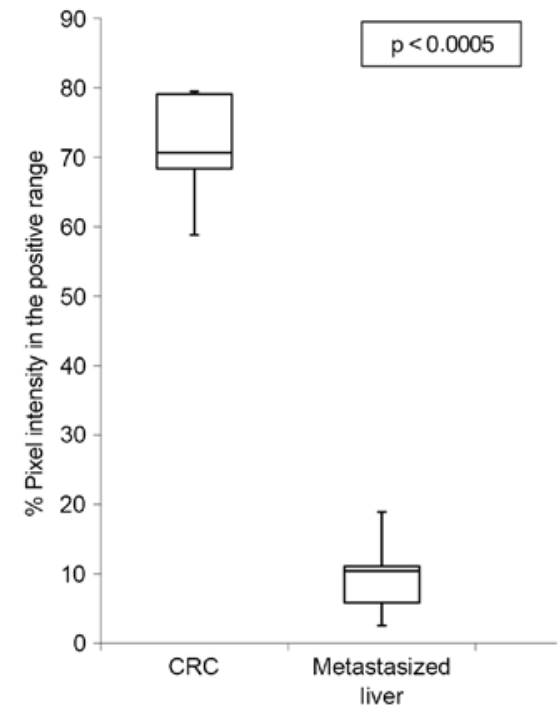

Figure 5. Box-and-whisker plot illustrating FKBP51 downregulation in metastasized liver tissue sections compared to FKBP51 expression in CRC tissue sections. Boxes indicate interquartile ranges, whiskers indicate ranges of maximal and minimal values. FKBP51, FK506-binding protein 5; CRC colorectal cancer.

pattern suggested mitochondrial localization of the protein in these cells.

FKBP51 protein is downregulated in metastasized liver tissue samples. Specimens were evaluated by two independent observers (a biologist and a pathologist) who were blinded to the conditions. In addition, ImageJ software and the open source plug-in IHC Profiler developed by Varghese et al (18) were used to compare the visual human interpretation to that of the computer-aided vision. Fig. 5 shows a box-and-whisker plot illustrating the results obtained using IHC Profiler to compare the percentage of positive pixels (pixel intensity range, 61-120) in the tissue samples. This clearly demonstrates the downregulation of FKBP51 protein in malignant liver specimens vs. CRC tissue samples $(7.5 \pm 4.3 \%$ in liver vs. $71.3 \pm 7.6 \%$ in $\mathrm{CRC}$; $\mathrm{P}<0.003)$.

No differences in distribution or in staining intensity were detected between samples from male or female patients, or among patients of different ages (data not shown).

\section{Discussion}

In a previous screening for biomarkers involved in oxaliplatin toxicity, FKBP5 was the gene whose transcriptional expression level was most downregulated quantitatively (5). In the current study, IHC analysis of FKBP51 protein expression and localization allowed observation of strong staining in the nuclei of enterocytes in healthy colon, whereas in colonic adenocarcinoma cells, the staining was localized in nuclei and cytoplasm. However, lesions exhibited variable staining, ranging from tumor nests with malignant cells strongly expressing FKBP51, to the surrounding stroma cells and lesions where no positive signal could be detected (Fig. 1E and F).

The observation that the expression of FKBP51 in tumor and stromal cells is associated with an immature phenotype of the surrounding stromal fibroblasts and with an increased microvessel density, as well as augmented tumor-associated macrophage infiltration, suggests a role for this protein in the epithelial-to-mesenchymal transition (EMT) process in CRC (20).

Patients in the current study received oxaliplatin-based chemotherapy prior to resection of the metastases. IHC analyses allowed the observation of changes in FKBP51 expression levels and localization in malignant liver compared with CRC. While in healthy liver FKBP51 protein exhibited strong staining in the nuclei of hepatocytes, in healthy regions of metastatic liver, the nuclear signal was fainter. The observed alterations in the liver tissue surrounding the metastases could be related to hepatic sinusoidal injury elicited by oxaliplatin therapy (21).

In liver metastases, the signal appeared faint or absent, while in the inflammatory fibrous stroma, several cells exhibited a strong signal (Fig. 3E). These structural changes could be associated with the effect of chemotherapy on tumor cells rather than with intrinsic changes of transformation of cells. This phenomenon is further supported by the fact that a patient with a predominantly negative immunostaining signal had a tumor (metastasis) that was completely resistant to chemotherapy. Lung metastases exhibited a similar expression pattern to liver metastases, with weak staining in tumor cells and a strong signal in inflammatory and fibrous stromal cells surrounding the metastases. Whether this weaker level of expression in the metastatic cells is related to chemotherapy or to their cell biology is to be determined by further studies.

FKBP51 and its related protein FKBP52 are HSP90 co-chaperones that influence steroid hormone receptor activity. These two immunophilins share a similar structure but act divergently due to differences in the FK1 domain and the proline-rich loop (13). Fig. 6 illustrates their coordinated functions. Due to differences in the FK1 domain, repression of hormone binding occurs in the presence of FKBP51, and potentiation in the presence of FKBP52. In the absence of a ligand, certain steroid hormone receptors reside primarily in the cytoplasm, whereas others are nuclear. Regardless of their primary localization, these receptors are not confined to any particular cell compartment, and instead shuttle continuously between the cytoplasm and nucleus (13). It is assumed that these signaling molecules move across the cell by simple diffusion. However, the fact that proteins of the HSP90-FKBP52 complex co-immunoprecipitate with the glucocorticoid and mineralocorticoid receptors, and with the dynein-dynactin complex (22-24), suggests that these motor proteins could power the retrograde movement of these steroid receptors. FKBP51 is generally considered to be a negative regulator of receptor function. Evidence suggests that it displays tissue- and/or cell-type-specific effects on receptor signaling (13). Furthermore, it has been reported that colorectal tumors produce glucocorticoids; these glucocorticoids would have immunosuppressive functions, leading to an increase in tumor survival and growth (25).

In transformed cells, the current results indicated a decrease in the expression levels of FKBP51, as observed in leukocytes. FKBP52 was not selected for analysis as no change in this gene was detected in the transcriptome analysis (5) or in the preliminary immunolocalization experiments. Fig. 6, which is based on the model of Storer et al (13), illustrates how a decrease in FKBP51 expression brings about the predominance or easier driving of the steroid receptor-hormone-HSP90-p23 complex to 




Figure 6. Model for FKBP51 interaction with steroid hormone receptor during translocation. Upon H binding, the SR heterocomplex exchanges FKBP51 for FKBP52, which is able to interact with dynein. The chaperone complex serves as a traction chain for the receptor through cytoskeletal tracts. The whole SR-chaperone complex translocates through the NPC. Receptor transformation is nucleoplasmic and facilitates binding of the steroid-activated receptor to promoter sites. Partially adapted from Storer et al (13). FKBP, FK506-binding protein; SR, steroid hormone receptor; H, steroid hormone; NPC, nuclear pore complex; Hsp90, heat shock protein 90.

the nucleus, making the transformed cell (at least after FOLFOX chemotherapy), somehow, more sensitive to steroid hormones. This fact needs to be further confirmed experimentally.

Baughman et al (26) reported in 1997 that FKBP51 is expressed in various tissues, but not in the colon, lung and spleen. In that study, the expression of FKBP51 was only analyzed by western blotting of protein lysate. However, in another study, in which different techniques were used (western blot, reverse transcription-polymerase chain reaction and IHC analyses), Mukaide et al (27) demonstrated that FKBP51 is expressed in normal epithelial cells and in adenocarcinoma cells in the human colon, and that there are no significant differences in the expression of FKBP51 between these cell types. Furthermore, the authors suggested that FKBP51 may suppress the proliferation of colorectal adenocarcinoma, possibly due to the suppression of function of the glucocorticoid receptors (27). The current findings only agree partially with these facts.

Previously, the RNA interference technique has been used to knock down the expression of FKBP5 in the A174 glioma cell line, revealing that FKBP5 expression aids in the regulation of glioma cell growth. By contrast, overexpression of FKBP5 markedly enhanced growth in this cell line (28). This fact agrees with the current results and explains a complementary effect of chemotherapy through FKBP pathways.

In summary, the present study supports the fundamental role of cell-by-cell IHC analysis in molecular data interpretation. The findings have demonstrated that the changes in
FKBP51 gene expression elicited by FOLFOX chemotherapy in PWCs of CRC patients can be confirmed at the protein level in tissue samples of colon adenocarcinoma prior to chemotherapy, compared with tissue sections of metastasized liver resected after oxaliplatin-based chemotherapy. Furthermore, the results indicated that, in CRC tissue sections, the expression of FKBP51 in tumor and stroma cells is associated with the immature phenotype of stromal fibroblasts and with the EMT phenotype, suggesting a role for this protein in the EMT process in CRC. The expression of FKBP51 in neural cells of the Auerbach's and Meissner's plexus could explain the development of oxaliplatin-induced autonomic neuropathy (29).

Finally, the observation that only certain cells in the tumor-associated stroma express FKBP51 must be further investigated to assess the hypothesis of a potential role for this immunophilin as a stromal cell subtype marker (30-32).

\section{Acknowledgements}

The authors would like to thank Dr Sonja Kennington for English language supervision and critical reading of the manuscript. This study was supported by Health Research Fund (FIS) PI11/00114 and FIS PI12/00729 grants, Spain. The study was also supported partially by the Insular Council of Tenerife and MCT-FEDER 2003/2004 (Olympus FV1000), and by an Improving Biomedical Research in the 
Canary Islands-7th Framework Program Research Potential (IMBRAIN-FP7-REGPOT)-2012-31637 grant.

\section{References}

1. Ferlay J, Shin HR, Bray F, Forman D, Mathers C and Parkin DM: Estimates of worldwide burden of cancer in 2008: GLOBOCAN 2008. Int J Cancer 127: 2893-2917, 2010.

2. Xu R, Zhou B, Fung PC and Li X: Recent advances in the treatment of colon cancer. Histol Histopathol 21: 867-872, 2006.

3. André T, Boni C, Mounedji-Boudiaf L, Navarro M, Tabernero J, Hickish T, Topham C, Zaninelli M, Clingan P, Bridgewater J, et al: Oxaliplatin, fluorouracil, and leucovorin as adjuvant treatment for colon cancer. N Engl J Med 350: 2343-2351, 2004.

4. Raymond E, Chaney SG, Taamma A and Cvitkovic E: Oxaliplatin: A review of preclinical and clinical studies. Ann Oncol 9: 1053-1071, 1998.

5. Morales M, Ávila J, González-Fernández R, Boronat L, Soriano ML and Martín-Vasallo P: Differential transcriptome profile of peripheral white cells to identify biomarkers involved in oxaliplatin induced neuropathy. J Pers Med 4: 282-296, 2014.

6. Erlejman AG, De Leo SA, Mazaira GI, Molinari AM, Camisay MF, Fontana V, Cox MB, Piwien-Pilipuk G and Galigniana MD: NF-kB transcriptional activity is modulated by FK506-binding proteins FKBP51 and FKBP52: A role for peptidyl-prolyl isomerase activity. J Biol Chem 289: 26263-26276, 2014.

7. Gallo LI, Lagadari M, Piwien-Pilipuk G and Galigniana MD: The 90-kDa heat-shock protein (Hsp90)-binding immunophilin FKBP51 is a mitochondrial protein that translocates to the nucleus to protect cells against oxidative stress. J Biol Chem 286: 30152-30160, 2011.

8. Li L, Lou Z and Wang L: The role of FKBP5 in cancer aetiology and chemoresistance. Br J Cancer 104: 19-23, 2011.

9. Pei H, Li L, Fridley BL, Jenkins GD, Kalari KR, Lingle W, Petersen G, Lou Z and Wang L: FKBP51 affects cancer cell response to chemotherapy by negatively regulating Akt. Cancer Cell 16: 259-266, 2009

10. Romano S, D'Angelillo A, Pacelli R, Staibano S, De Luna E, Bisogni R, Eskelinen EL, Mascolo M, Cali G, Arra C and Romano MF: Role of FK506-binding protein 51 in the control of apoptosis of irradiated melanoma cells. Cell Death Differ 17: 145-157, 2010.

11. Romano S, Staibano S, Greco A, Brunetti A, Nappo G, Ilardi G, Martinelli R, Sorrentino A, Di Pace A, Mascolo M, et al: FK506 binding protein 51 positively regulates melanoma stemness and metastatic potential. Cell Death Dis 4: e578, 2013.

12. Romano S, D'Angelillo A, D'Arrigo P, Staibano S, Greco A, Brunetti A, Scalvenzi M, Bisogni R, Scala I and Romano MF: FKBP51 increases the tumour-promoter potential of TGF-beta. Clin Transl Med 3: 1, 2014.

13. Storer CL, Dickey CA, Galigniana MD, Rein T and Cox MB: FKBP51 and FKBP52 in signaling and disease. Trends Endocrinol Metab 22: 481-490, 2011

14. Hubler TR, Denny WB, Valentine DL, Cheung-Flynn J, Smith DF and Scammell JG: The FK506-binding immunophilin FKBP51 is transcriptionally regulated by progestin and attenuates progestin responsiveness. Endocrinology 144: 2380-2387, 2003.

15. Jinwal UK, Koren J III, Borysov SI, Schmid AB, Abisambra JF, Blair LJ, Johnson AG, Jones JR, Shults CL, O'Leary JC III, et al: The Hsp90 cochaperone, FKBP51, increases Tau stability and polymerizes microtubules. J Neurosci 30: 591-599, 2010.
16. Chambraud B, Sardin E, Giustiniani J, Dounane O, Schumacher M, Goedert M and Baulieu EE: A role for FKBP52 in Tau protein function. Proc Natl Acad Sci USA 107: 2658-2663, 2010.

17. Li L, Fridley B, Kalari K, Jenkins G, Batzler A, Safgren S, Hildebrandt M, Ames M, Schaid D and Wang L: Gemcitabine and cytosine arabinoside cytotoxicity: Association with lymphoblastoid cell expression. Cancer Res 68: 7050-7058, 2008.

18. Varghese F, Bukhari AB, Malhotra R and De A: IHC Profiler: An open source plugin for the quantitative evaluation and automated scoring of immunohistochemistry images of human tissue samples. PLoS One 9: e96801, 2014.

19. Bleau AM, Agliano A, Larzabal L, de Aberasturi AL and Calvo A: Metastatic dormancy: A complex network between cancer stem cells and their microenvironment. Histol Histopathol 29: 1499-1510, 2014.

20. Ha SY, Yeo SY, Xuan Y and Kim SH: The prognostic significance of cancer-associated fibroblasts in esophageal squamous cell carcinoma. PLoS One 9: e99955, 2014.

21. Nalbantoglu IL, Tan BR Jr, Linehan DC, Gao F and Brunt EM: Histological features and severity of oxaliplatin-induced liver injury and clinical associations. J Dig Dis 15: 553-560, 2014.

22. Davies TH, Ning YM and Sánchez ER: A new first step in activation of steroid receptors: Hormone-induced switching of FKBP51 and FKBP52 immunophilins. J Biol Chem 277: 4597-4600, 2002.

23. Galigniana MD, Radanyi C, Renoir JM, Housley PR and Pratt WB: Evidence that the peptidylprolyl isomerase domain of the hsp90-binding immunophilin FKBP52 is involved in both dynein interaction and glucocorticoid receptor movement to the nucleus. J Biol Chem 276: 14884-14889, 2001.

24. Wochnik GM, Rüegg J, Abel GA, Schmidt U, Holsboer F and Rein T: FK506-binding proteins 51 and 52 differentially regulate dynein interaction and nuclear translocation of the glucocorticoid receptor in mammalian cells. J Biol Chem 280: 4609-4616, 2005.

25. Sidler D, Renzulli P, Schnoz C, Berger B, Schneider-Jakob S, Flück C, Inderbitzin D, Corazza N, Candinas D and Brunner T: Colon cancer cells produce immunoregulatory glucocorticoids. Oncogene 30: 2411-2419, 2011.

26. Baughman G, Wiederrecht GJ, Chang F, Martin MM and Bourgeois S: Tissue distribution and abundance of human FKBP51, and FK506-binding protein that can mediate calcineurin inhibition. Biochem Biophys Res Commun 232: 437-443, 1997.

27. Mukaide H, Adachi Y, Taketani S, Iwasaki M, Koike-Kiriyama N, Shigematsu A, Shi M, Yanai S, Yoshioka K, Kamiyama Y and Ikehara S: FKBP51 expressed by both normal epithelial cells and adenocarcinoma of colon suppresses proliferation of colorectal adenocarcinoma. Cancer Invest 26: 385-390, 2008.

28. Jiang W, Cazacu S, Xiang C, Zenklusen JC, Fine HA, Berens M, Armstrong B, Brodie C and Mikkelsen T: FK506 binding protein mediates glioma cell growth and sensitivity to rapamycin treatment by regulating NF-kappaB signaling pathway. Neoplasia 10 : 235-243, 2008.

29. Vandamme M, Pauwels W and Bleecker JD: A case of delayed oxaliplatin-induced pseudo-obstruction: An atypical presentation of oxaliplatin neurotoxicity. Acta Clin Belg 70: 207-210, 2015.

30. Calon A, Lonardo E, Berenguer-Llergo A, Espinet E, Hernando-Momblona X, Iglesias M, Sevillano M,Palomo-Ponce S, Tauriello DV, Byrom D, et al: Stromal gene expression defines poor-prognosis subtypes in colorectal cancer. Nat Genet 10: 320-329, 2015.

31. Kuroda N,Nakayama H, Miyazaki E, Toi M, Hiroi M and Enzan H: The distribution of CD34-positive stromal cells and myofibroblasts in colorectal carcinoid tumors. Histol Histopathol 20: 27-33, 2005.

32. Sugimoto H, Mundel TM, Kieran MW and Kalluri R: Identification of fibroblast heterogeneity in the tumor microenvironment. Cancer Biol Ther 5: 1640-1646, 2006. 